\title{
TWO WEIGHT INEQUALITIES FOR INDIVIDUAL HAAR MULTIPLIERS AND OTHER WELL LOCALIZED OPERATORS
}

\author{
F. Nazarov, S. Treil, and A. VolberG
}

\begin{abstract}
In this paper we are proving that Sawyer type condition for boundedness work for the two weight estimates of individual Haar multipliers, as well as for the Haar shift and other "well localized" operators.
\end{abstract}

\section{Introduction}

The main question of this paper concerns two weight estimates for singular integral operators, i.e. the questions when an integral operator $T$ is bounded operator from a weighted space $L^{2}(w)$ to $L^{2}(v)$.

One of the most interesting cases is the case when $T$ is the Hilbert transform, $T f(s)=\frac{1}{\pi} \int \frac{f(t)}{s-t} d t$, although the case of more general Calderón-Zygmund operators seems to be of great interest as well. For such operators no "real variable" necessary and sufficient condition is known. ${ }^{1}$

In this paper we deal with dyadic analogues of the Hilbert transform, the so-called Haar multipliers and their generalizations. It turns out that for such operators it is possible to find necessary and sufficient condition for two weight estimates.

Let us introduce one of the main examples. The standard dyadic grid $\mathcal{D}=\mathcal{D}_{0}$ is the collection of all dyadic intervals $\left[2^{k} \cdot j, 2^{k} \cdot(j+1)\right), k, j \in \mathbb{Z}$. A general dyadic grid is an object obtained from $\mathcal{D}_{0}$ by a dilation and a shift.

For an interval $I \subset \mathbb{R}$ we define the $\left(L^{2}\right.$-normalized) Haar function

$$
h_{I}:=|I|^{-1 / 2}\left(\chi_{I_{+}}-\chi_{I_{-}}\right):
$$

here $|I|$ stands for the length of the interval $I ; I_{+}$and $I_{-}$denote its right and left halves respectively, and $\chi_{E}$ denotes the characteristic function (indicator) of the set E.

Received by the editors February 1, 2007.

This paper is based upon work supported by the National Science Foundation under Grants DMS-0501065, DMS-0501067.

${ }^{1}$ For the Hilbert transform a necessary and sufficient condition of Helson-Szegö type was obtained by M. Cotlar and C. Sadosky [CS1]. Their condition was stated in the language of complex analysis: the Hilbert transform is a bounded operator from $L^{2}(w) \rightarrow L^{2}(v)$ if and only if one can find a function $h$ in the analytic Hardy class $H^{1}$ such that for some $C>0$ the matrix $\left(\begin{array}{cc}C w-v & C w+v-h \\ C w+v-\bar{h} & C w-v\end{array}\right)$ is positive semidefinite a.e. No generalization of this condition to general Calderón-Zygmund operators is known. 
Given a sequence $\alpha=\left\{\alpha_{I}\right\}_{I \in \mathcal{D}}$ define the Haar multiplier (a.k.a. the martingale transform) $T^{\alpha}$ by

$$
T^{\alpha} f:=\sum_{I \in \mathcal{D}} \alpha_{I}\left(f, h_{I}\right) h_{I}, \quad f \in L^{2}(\mathbb{R}) .
$$

We are interested under what conditions on the weights $v, w\left(v, w \geq 0, v, w \in L_{\text {loc }}^{1}\right)$ the operator $T=T^{\alpha}$ extends to a bounded operator from $L^{2}(w)$ to $L^{2}(v)$, i.e., when the following two weight estimate

$$
\int_{\mathbb{R}}\left|T^{\alpha} f\right|^{2} v d x \leq C \int_{\mathbb{R}}|f|^{2} w d x
$$

holds.

If one assumes that $w^{-1} \in L_{\text {loc }}^{1}$ (this assumption is satisfied in practically all interesting cases), then the above estimate means that the operator $M_{v^{1 / 2}} T^{\alpha} M_{w^{-1 / 2}}$, where $M_{\phi}$ stands for the operator of multiplication by $\phi$, is bounded in the nonweighted $L^{2}$ space.

In [NTV1] this question was studied for the family of Haar multipliers $T^{\sigma \alpha}$, where $\alpha=\left\{\alpha_{I}\right\}_{I \in \mathcal{D}}$ is fixed and $\sigma=\left\{\sigma_{I}\right\}_{I \in \mathcal{D}}$ is an arbitrary sequence of signs, $\sigma_{I}= \pm 1$. It was shown that the operators $M_{v^{1 / 2}} T^{\sigma \alpha} M_{w^{-1 / 2}}$ are uniformly (over all possible choices of signs $\sigma$ ) bounded if and only if the operators are uniformly bounded on the test functions $w^{-1 / 2} \chi_{I}, I \in \mathcal{D}$, and their adjoints $M_{w^{-1 / 2}} T^{\sigma \alpha} M_{v^{1 / 2}}$ are uniformly bounded on the test functions $v^{1 / 2} \chi_{I}, I \in \mathcal{D}$.

Conditions where the boundedness of an operator follows from its boundedness on special test functions as above are called "Sawyer type conditions", after E. Sawyer, who proved in [S1] that such condition is necessary and sufficient for two weight estimates of the maximal function (only one condition is needed there). After that there were quite a few results that Sawyer type conditions are sufficient for the boundedness of (clearly they are necessary) of different classes of integral operators with positive kernels.

The above mentioned result about Haar multipliers was a first (and up until now a unique) result giving necessary and sufficient condition for the boundedness of operators with alternating kernels. The fact that helped us a lot was that we were dealing with a family of operators, and that allowed us to reduce the problem to estimates of an operator with non-negative kernel.

At that moment it seemed impossible to get a sharp result about two weight estimates for an individual Haar multiplier, at least our method did not allow us to do that.

So, the main result of the paper looks quite surprising: the Sawyer type condition works for two weight estimates of an individual Haar multiplier (as well as for a wider class of so-called "band operators")!

Before giving a formal definition, let us present one more important example of an "band operator", the so called Haar Shift $\mathcal{S}$

$$
\mathcal{S} f:=\sum_{I \in \mathcal{D}}\left(f, h_{I}\right)\left[h_{I_{+}}-h_{I_{-}}\right] .
$$

This operator is interesting, in particular, because if we average it over all dyadic grids (translated and rescaled) we get the Hilbert transform (up to a multiplicative constant), see $[\mathrm{P}]$. 


\section{Two weight estimates of band operators: formal definitions and main results}

1.1. Main definitions. Let $\mathcal{D}$ be the standard dyadic lattice in $\mathbb{R}^{N}$. Let us recall the definition: for each $k \in \mathbb{Z}$ we consider the cube $\left[0,2^{k}\right)^{N}$ and all its translations by elements of $\mathbb{R}^{N}$ with coordinates of form $j \cdot 2^{k}, j \in \mathbb{Z}$, then take union over all $k$. Each dyadic cube has $2^{N}$ "sons", and each cube has a unique "parent".

In this paper by a cube we will always mean a dyadic cube.

For a cube $Q$ let $\ell(Q)$ denote its size, i.e. the length of its side. And for a cube $Q$ let $Q^{(k)}$ be the $k$ th grandparent of the cube $Q$, i.e. the cube $R$ of size $2^{k} \ell(Q)$ containing $Q$.

A (non-weighted) Haar function on a cube $Q$ is a function $h_{Q}$ supported on $Q$, constant on the "children" of $Q$ and orthogonal to constants, $\int_{Q} h_{Q} d x=0$. The set of all Haar function on a cube $Q \subset \mathbb{R}^{N}$ is a vector space of dimension $2^{N}-1$.

With a dyadic lattice $\mathcal{D}$ in $\mathbb{R}^{N}$ one can naturally associate a (non-oriented) $2^{N}$-ary tree, where each dyadic cube is connected to its $2^{N}$ "sons". By the "tree distance" (or "graph distance") $d_{\text {tree }}(Q, R)$ between cubes $Q, R \in \mathcal{D}$ we understand the distance on the graph, where we assign length 1 to each wedge.

And now the formal definition:

Definition 1.1. A band operator on $\mathbb{R}^{N}$ is a bounded operator in $L^{2}\left(\mathbb{R}^{d}\right)$ whose matrix in the Haar basis has the "band structure", meaning there exists $r \in \mathbb{Z}_{+}$such that $\left(T h_{Q}, h_{R}\right)=0$ for all Haar functions $h_{Q}, h_{R}$ such that $d_{\text {tree }}(Q, R)>r$.

The Haar multipliers (martingale transforms) discussed in the introductions are band operators with $r=0$, and the Haar shift is a band operator with $r=1$.

1.2. Two weigh estimates. As we already mentioned above, the operator $T$ can be extended to a bounded operator acting from $L^{2}(w)$ to $L^{2}(v)$ if and only if the operator $M_{v}^{1 / 2} T M_{w}^{-1 / 2}$ can be extended to a bounded operator in the (non-weighted) $L^{2}$. Denoting $u:=w^{-1}$ we can write the problem in more symmetric form, namely, when the operator $M_{v}^{1 / 2} T M_{u}^{1 / 2}$ can be extended to a bounded operator in the nonweighted $L^{2}$ ? Here we assume that $u$ and $v$ are $L_{\text {loc }}^{1}$ weights. This formulation is (at least formally) a bit more general then the formulation with two weight estimates, because here we do not assume that $u^{-1} \in L_{\text {loc }}^{1}$ (and we did assume above that $\left.w^{-1} \in L_{\mathrm{loc}}^{1}\right)$.

Now we are ready to state one of the main results of the paper:

Theorem 1.2. Let $T$ be a band operator, and let $u, v \geq 0, u, v \in L_{l o c}^{1}$. Then the operator $M_{v}^{1 / 2} T M_{u}^{1 / 2}$ extends to a bounded operator in $L^{2}$ if and only if for all dyadic cubes $Q$ the following two inequalities hold:

$$
\begin{gathered}
\int_{\mathbb{R}^{N}}\left|T\left(\chi_{Q} u\right)\right|^{2} v d x \leq C \int_{Q} u d x, \\
\int_{\mathbb{R}^{N}}\left|T^{*}\left(\chi_{Q} v\right)\right|^{2} u d x \leq C \int_{Q} v d x .
\end{gathered}
$$


Moreover, the norm of the operator $M_{v}^{1 / 2} T M_{u}^{1 / 2}$ can be estimated by a constant depending only on the dimension $N$, the number $r$ in the definition of the band matrix, and the constants $C$ in (1.1), (1.2)

The above theorem says that to verify the boundedness of the operator $M_{v}^{1 / 2} T M_{u}^{1 / 2}$ it is sufficient only to check its boundedness on the test functions $u^{1 /} \chi_{Q}$ and the boundedness of its formal adjoint $M_{u}^{1 / 2} T^{*} M_{v}^{1 / 2}$ on the test functions $v^{1 / 2} \chi_{Q}$ for all dyadic cubes $Q$.

Remark 1.3. A careful reader can ask a question here on how one can interpret the expressions $T\left(\chi_{Q} u\right)$ and $T^{*}\left(\chi_{Q} v\right)$. The problem is that the operator $T$ (and so its adjoint $\left.T^{*}\right)$ are defined only on $L^{2}$, so if one only assumes that $u, v \in L_{\mathrm{loc}}^{1}$, the above expressions formally are not defined.

However, it is easy to give the meaning to the conditions (1.1), (1.2). First of all, $T\left(\chi_{Q} u\right)$ and $T^{*}\left(\chi_{Q} v\right)$ are well defined if $u, v \in L_{\text {loc }}^{2}$. Second, it is trivial, that for any weight $v^{\prime} \leq v$ we have $\left\|M_{v^{\prime}}^{1 / 2} T M_{u}^{1 /}\right\| \leq\left\|M_{v}^{1 / 2} T M_{u}^{1 /}\right\|$, and by taking adjoint one can conclude that for any weight $u^{\prime} \leq u$ we have $\left\|M_{v}^{1 / 2} T M_{u^{\prime}}^{1 /}\right\| \leq\left\|M_{v}^{1 / 2} T M_{u}^{1 /}\right\|$.

Therefore, for any sequence of weights $u_{n}, u_{n} \in L^{2}(Q), u_{n} \nearrow u$, the expression $T u_{n} \chi_{Q}$ is well defined. If, in addition, one assumes that the operator $M_{v}^{1 / 2} T M_{u}^{1 / 2}$ is bounded, then $\left\|v^{1 / 2} T u_{n} \chi_{Q}\right\|_{L^{2}} \leq C<\infty$, and moreover $v^{1 / 2} T u_{n} \chi_{Q}$ converges to some functions in $L^{2}$. It is easy to see that this limit function does not depend on the choice of the sequence $u_{n}$, so we call this function $v^{1 / 2} T \chi_{Q} u$. The right side of (1.1) then can be interpreted as the $L^{2}$ norm of this function. The dual condition (1.2) can be interpreted similarly.

Remark 1.4. For the sufficiency part one can think even of a simpler condition to interpret (1.1), (1.2). For example, one can pick an increasing sequence of weights $u_{n} \in L_{\mathrm{loc}}^{2} \nearrow u$ (for example $\left.u_{n}(x)=\min \{u(x), n\}\right)$ and interpret the condition (1.1) as the uniform estimate (independent of $n$ and $Q$ )

$$
\int_{\mathbb{R}^{N}}\left|T\left(\chi_{Q} u_{n}\right)\right|^{2} v d x \leq C \int_{Q} u d x
$$

The dual condition (1.2) is interpreted similarly by picking an increasing sequence $v_{n} \nearrow v$.

Indeed, these conditions would imply the conditions (1.1), (1.2) with $u$ and $v$ replaced by $u_{n}$ and $v_{m}$ respectively. That, by Theorem (1.2) implies the uniform estimate $\left\|M_{v_{n}}^{1 / 2} T M_{u_{m}}^{1 / 2}\right\| \leq C<\infty$ which implies the estimate $\left\|M_{v}^{1 / 2} T M_{u}^{1 / 2}\right\| \leq C$.

The conditions of Theorem 1.2 can be relaxed a bit by integrating only over the cubes $Q$ :

Theorem 1.5. Let $T$ be a band operator and let $u, v \geq 0, u, v \in L_{\text {loc }}^{1}$. Then the operator $M_{v}^{1 / 2} T M_{u}^{1 / 2}$ extends to a bounded operator in $L^{2}$ if and only if for all dyadic cubes $Q$ the following two conditions hold:

(1) For all dyadic cubes $Q, R$ satisfying $2^{-r} \ell(Q) \leq \ell(R) \leq 2^{r} \ell(Q)$

$$
\left|\left\langle T \chi_{Q}, \chi_{R}\right\rangle\right| \leq C\left(\int_{Q} u\right)^{1 / 2}\left(\int_{Q} v\right)^{1 / 2}
$$


(2) For all dyadic cubes $Q$

$$
\int_{\mathbb{R}^{N}}\left|T\left(\chi_{Q} u\right)\right|^{2} v d x \leq C \int_{Q} u d x, \quad \int_{\mathbb{R}^{N}}\left|T^{*}\left(\chi_{Q} v\right)\right|^{2} u d x \leq C \int_{Q} v d x .
$$

Moreover, the norm of the operator $M_{v}^{1 / 2} T M_{u}^{1 / 2}$ can be estimated by a constant depending only on the dimension $N$, the number $r$ in the definition of the band matrix, and the constants $C$ in the above conditions (1) and (2).

In this paper $\langle\cdot, \cdot\rangle$ stands for the standard inner product in $L^{2}\left(\mathbb{R}^{N}\right),\langle f, g\rangle=$ $\int f \bar{g} d x$. Note that

$$
\left\langle T \chi_{Q} u, \chi_{R} v\right\rangle=\left\langle M_{v}^{1 / 2} T M_{u}^{1 / 2} \chi_{Q} u^{1 / 2}, \chi_{R} v^{1 / 2}\right\rangle,
$$

so, as we discussed above in Remark 1.3, this expression is well defined.

Remark 1.6. Note, that in fact one does not even need the operator $T$ to be bounded in $L^{2}$. A bit more elaborate reasoning than in Remark 1.3 would allow us to interpret the conditions of Theorem 1.2 in the case when we only require the bilinear form $\langle T f, g\rangle$ of the operator $T$ be defined on bounded compactly supported functions $f$ and $g$. We leave the details here as an exercise for the reader.

\section{Well-localized operators for general measures}

To prove theorems 1.2 and 1.5 we prove even a bit more general results about the so-called well-localized operators $T=T_{\mu}: L^{2}(\mu) \rightarrow L^{2}(\nu)$ for general measures $\mu, \nu$ that may have singular parts.

2.1. Heuristics and formal definition. The idea behind the notion of well-localized operators is rather simple. If one has an integral operator $T$,

$$
T f(x)=\int K(x, y) f(y) d m(y),
$$

where the integration is with respect to the Lebesgue measure $m$ in $\mathbb{R}^{N}$, one can consider (at least formally) the operator $T_{\mu}$ where the integration with respect to the measure $\mu$,

$$
T_{\mu} f(x)=\int K(x, y) f(y) d \mu(y) .
$$

Such reduction is quite common in weighted estimates. For example, two weight estimates for integral operators $T$ can be reduced to estimates of operators $T_{\mu}$ : $L^{2}(\mu) \rightarrow L^{2}(\nu)$ with appropriately chosen measures $\mu$ and $\nu$. One of the advantages of such representation is that the integration in the operator and in the computation of the norm is with respect to the same measure $\mu$.

Since a rank one operator $\langle\cdot, f\rangle g$ is an integral operator with kernel $g(x) \overline{f(y)}$, one can formally represent any operator $T$ is $L^{2}$ as an integral operator by considering its matrix in an orthonormal basis (for example in the Haar basis) and writing it as a sum of rank one operators. And then one can replace the integration with respect to the Lebesgue measure by the integration with respect to the measure $\mu$. However this is only a formal reasoning, because, first of all, the resulting "kernel" of the "integral" operator does not need to be a function. And even if the kernel $K$ is a function, it is completely not clear how to interpret the operator $T_{\mu}$. 
So, instead of writing a band operator as an integral operator (which is not always possible: for example, the identity $I$ is a band operator) and then trying to interpret the operator $T_{\mu}$, we pick an axiomatic approach.

Looking at the above formal reasoning, one can figure out what structure the matrix of the operator $T_{\mu}$ should have with respect to the weighted Haar bases in $L^{2}(\mu)$ and $L^{2}(\nu)$, if one start with a band operator $T$. It is not difficult to see, that while the matrix of $T_{\mu}$ in the weighted Haar bases is not generally a band matrix, it has some special properties, some traces of the band structure of $T$.

So, we took this special structure of the matrix in the weighted Haar bases as the definition of the well-localized operators. Later in this section we will show how for a band operator $T$ one can rigorously reduce the estimates of the operator $M_{v}^{1 / 2} T M_{u}^{1 / 2}$ to the estimates of of the appropriate well-localized operator $T_{\mu}$

And now main definitions.

First, let us define the weighted Haar system. For each cube $Q$ a Haar function with respect to a measure $\mu$ ( $\mu$-Haar function) on $Q$ is a function $h_{Q}^{\mu}$ supported on $Q$, constant on all $2^{N}$ "children" of $Q$, and such that $\int_{Q} h_{Q}^{\mu} d \mu=0$. Note that for a given $Q$ the set $H_{Q}^{\mu}$ of all $\mu$-Haar functions on $Q$ is a subspace of dimension at most $2^{N}-1$ (can be less since degenerate situations are possible). The set $H_{Q}$ of non-weighted Haar functions on $Q$ has exactly dimension $2^{N}-1$.

Let $\mu$ and $\nu$ are (regular Borel) measures in $\mathbb{R}^{N}$. Let us be given an operator $T=T_{\mu}$ acting from $L^{2}(\mu)$ to $L^{2}(\nu)$. By given we mean that we know its bilinear form $\left\langle T \chi_{Q}, \chi_{R}\right\rangle_{\nu}$ on characteristic functions of cubes; here $\langle\cdot, \cdot\rangle_{\nu}$ is the inner product in $L^{2}(\nu)$,

$$
\langle f, g\rangle_{\nu}=\int f \bar{g} d \nu
$$

Note that the above bilinear forms also define a formal adjoint $T^{*}=T_{\nu}^{*}$ of $T=T_{\mu}$.

Definition 2.1. Let $T=T_{\mu}$ be he operator defined above, acting (formally) from $L^{2}(\mu)$ to $L^{2}(\nu)$. We say that $T_{\mu}$ is lower triangularly localized if there exists a constant $r>0$ such that for all cubes $R$ and $Q, \ell(R) \leq \ell(Q)$, and for all $\nu$-Haar functions $h_{R}^{\mu}$ on $R$

$$
\left\langle T_{\mu} \chi_{Q}, h_{R}^{\nu}\right\rangle_{\nu}=0
$$

if $R \not \subset Q^{(r)}$ or if $\ell(R) \leq 2^{-r} \ell(Q)$ and $R \not \subset Q$. Here, recall, $Q^{(r)}$ is the "grandparent" of order $r$ of the cube $Q$.

And we say that the operator $T_{\mu}$ is well localized if both $T_{\mu}$ and its formal adjoint $T_{\nu}^{*}$ are lower triangularly localized.

Note, that the Haar multipliers and Haar shift, discussed in the Introduction are well localized in the sense of the above definition, see Section 2.2 below. Note, that while the matrix of a Haar multiplier $T^{\alpha}$ in the non-weighted case has only one diagonal, the matrix of the weighted version may have infinitely many.

2.2. From weighted estimates for band operators to estimates of well localized operators. First we want to show that two weight estimates for the band operators can be reduced from the estimates for the well-localized operators. 
Let $T$ be a band operator in the non-weighted $L^{2}\left(\mathbb{R}^{N}\right)$ and let $u, v \geq 0, u, v \in L_{\text {loc }}^{1}$ be two weights. Suppose we want to know whether the operator $M_{v}^{1 / 2} T M_{u}^{1 / 2}$ is bounded.

Let us denote $d \nu=v d m, d \mu=u d m$, where $m$ is the Lebesgue measure in $\mathbb{R}^{N}$. Note that $M_{u^{1 / 2}}: L^{2}(\mu) \rightarrow L^{2}(m), M_{v^{1 / 2}}: L^{2}(\nu) \rightarrow L^{2}(m)$ are isometries. So the boundedness of the operator $M_{v}^{1 / 2} T M_{u}^{1 / 2}$ in $L^{2}(m)$ is equivalent to the boundedness of the operator

$$
T_{\mu}=M_{v^{-1 / 2}}\left(M_{v}^{1 / 2} T M_{u}^{1 / 2}\right) M_{u^{1 / 2}}=T M_{u}
$$

acting from $L^{2}(\mu)$ to $L^{2}(\nu)$.

If the operator $T$ is an integral operator

$$
T f(x)=\int_{\mathbb{R}^{N}} K(x, y) f(y) d m(y),
$$

then the operator $T_{\mu}$ is the integral operator with the same kernel $K$, but the integration is performed with respect to the measure $d \mu=u d m$,

$$
T_{\mu} f(x)=\int_{\mathbb{R}^{N}} K(x, y) f(y) d \mu(y) .
$$

The adjoint of the operator $T_{\mu}: L^{2}(\mu) \rightarrow L^{2}(\nu)$ is the operator $T_{\nu}^{*}: L^{2}(\nu) \rightarrow L^{2}(\mu)$,

$$
T_{\nu}^{*}=T^{*} M_{v} \text {. }
$$

Again, in the case of integral operator, we have the following representation of $T_{\nu}^{*}$.

$$
T_{\nu}^{*} g(y)=\int_{\mathbb{R}^{N}} \overline{K(x, y)} g(x) d \nu(x),
$$

which explains the subscript $\nu$.

Remark. The formula $T_{\nu}^{*}=T^{*} M_{v}$ may seem strange, it looks like the formula should be $M_{u} T^{*}$. However, this is a correct formula, and the naturally looking formula $M_{u} T^{*}$ is wrong, the main reason being that we are considering operators acting between different spaces.

Namely, if we represent $T_{\mu}$ as

$$
T_{\mu}=M_{v^{-1 / 2}}\left[M_{v^{1 / 2}} T M_{u^{1 / 2}}\right] M_{u^{1 / 2}}
$$

then in brackets all operators are operators in $L^{2}$, so multiplication operators are selfadjoint ones. But outside the brackets, the operators $M_{v^{-1 / 2}}$ and $M_{u^{1 / 2}}$ are unitary operators, $M_{v^{-1 / 2}}: \operatorname{Ran} M_{v^{1 / 2}} \subset L^{2} \rightarrow L^{2}(\nu)$ and $M_{u^{1 / 2}}: L^{2}(\mu) \rightarrow \operatorname{Ran} M_{u^{1 / 2}} \subset L^{2}$. So their adjoint are their inverses, and so

$$
\left(T_{\mu}\right)^{*}=M_{u^{-1 / 2}}\left[M_{u^{1 / 2}} T^{*} M_{v^{1 / 2}}\right] M_{v}^{1 / 2}=T^{*} M_{v}=: T_{\nu}^{*} .
$$

Let us now show that the operator $T_{\mu}$ is indeed a well localized operator in the sense of Definition 2.1.

Consider a decomposition of the operator $T$ with respect to the non-weighted Haar basis in $L^{2}(m)$,

$$
T=\sum_{R, Q \in \mathcal{D}} T_{R, Q}, \quad T_{R, Q}: H_{Q} \rightarrow H_{R}
$$

here recall that $H_{Q}$ denotes the space of all non-weighted Haar functions $h_{Q}$ on the cube $Q$ (which is a subspace of $L^{2}(m)$ of dimension $2^{N}-1$ ). If we chose some 
orthonormal bases $\left\{h_{Q, k}\right\}_{k=1}^{2^{N}-1}$ and $\left\{h_{R, j}\right\}_{j=1}^{2^{N}-1}$ in $H_{Q}$ and $H_{R}$ respectively, then we can represent $T_{R, Q}$ as

$$
T_{R, Q}=\sum_{j, k=1}^{2^{N}-1}\left\langle T h_{Q, k}, h_{R, j}\right\rangle\left\langle\cdot, h_{Q, k}\right\rangle h_{R, j} .
$$

Since the rank one operator $\left\langle\cdot, h_{Q, k}\right\rangle_{R, j}$ is an integral operator with kernel $\overline{h_{Q, k}(y)} h_{R, j}(x)$, we can conclude that $T_{R, Q}$ can be represented as an integral operator with bounded compactly supported kernel. So, the operators $T_{R, Q}$ are well defined on $L_{\mathrm{loc}}^{1}$ functions.

Assume for a moment that $u \in L_{\text {loc }}^{2}$. Then $u \chi_{Q} \in L^{2}$, so

$$
T u \chi_{Q}=\sum_{Q^{\prime}, R^{\prime}} T_{R^{\prime}, Q^{\prime}} u \chi_{Q}
$$

where the series converges in $L^{2}$. Consider a cube $R, \ell(R) \leq \ell(Q)$. If we also assume that $v \in L_{\text {loc }}^{2}$, then $\left\langle T u \chi_{Q}, h_{R}^{\nu}\right\rangle_{\nu}=\left\langle T u \chi_{Q}, v h_{R}^{\nu}\right\rangle$ is well defined and

$$
\left\langle T u \chi_{Q}, h_{R}^{\nu}\right\rangle_{\nu}=\sum_{Q^{\prime}, R^{\prime} \in \mathcal{D}}\left\langle T_{R^{\prime}, Q^{\prime}} \chi_{Q} u, h_{R}^{\nu}\right\rangle_{\nu}
$$

Note, that $T_{R^{\prime}, Q^{\prime}} \chi_{Q} u \neq 0$ only if $Q^{\prime} \cap Q \neq \varnothing$, so in the above sum we need to consider only such $Q^{\prime}$. Since a weighted Haar function $h_{R}^{\nu}$ is orthogonal in $L^{2}(\nu)$ to constants, $\left\langle T_{R^{\prime}, Q^{\prime}} \chi_{Q} u, h^{\nu}\right\rangle_{\nu}=0$ for $R \varsubsetneqq R^{\prime}$. Also, trivially $\left\langle T_{R^{\prime}, Q^{\prime}} \chi_{Q} u, h^{\nu}\right\rangle_{\nu}=0$ if $R^{\prime} \cap R \neq \varnothing$. And finally, the band structure of $T$ means that $T_{R^{\prime}, Q^{\prime}}=0$ if $d_{\text {tree }}\left(R^{\prime}, Q^{\prime}\right)>r$. So in the above sum we can consider only $R^{\prime}, Q^{\prime}$ satisfying $Q^{\prime} \cap Q \neq \varnothing, R^{\prime} \subset R$ and $d_{\text {tree }}\left(R^{\prime}, Q^{\prime}\right) \leq r$.

Since $R^{\prime} \subset R$ and $\ell(R) \leq \ell(Q)$, the assumption $\mathrm{d}_{\text {tree }}\left(R^{\prime}, Q^{\prime}\right) \leq r$ implies that $\ell\left(Q^{\prime}\right) \leq 2^{r} \ell(Q)$. This together with $Q \cap Q^{\prime} \neq \varnothing$ implies that $Q^{\prime} \subset Q^{(r)}$. Indeed, $Q \cap Q^{\prime} \neq \varnothing$ means that either $Q^{\prime} \subset Q$ (and so $\left.Q^{\prime} \subset Q^{(r)}\right)$, or $Q \subset Q^{\prime}$, and in the latter case the estimate on the size of $Q^{\prime}$ implies $Q^{\prime} \subset Q^{(r)}$.

Note that if $R \not \subset Q^{(r)}$ then $R^{\prime} \not \subset Q^{(r)}$ (because $\left.R^{\prime} \subset R\right)$. Therefore, $\mathrm{d}_{\text {tree }}\left(R^{\prime}, Q^{\prime}\right) \geq$ $r+2$, because we need at least one step to go above $Q^{(r)}$ and then at least $r+1$ steps to go to cubes of size $\ell(R)$. Therefore, $\left\langle T\left(w^{-1} \chi_{Q}\right), h_{R}^{\nu}\right\rangle_{\nu}=0$ if $R \not \subset Q^{(r)}$.

A similar reasoning works for the case $\ell(R) \leq 2^{-r} \ell(Q)$. Namely, the inclusion $R^{\prime} \subset R$ and the inequality $\mathrm{d}_{\text {tree }}\left(R^{\prime}, Q^{\prime}\right) \leq r$ imply that $\ell\left(Q^{\prime}\right) \leq \ell(Q)$, so it follows from $Q \cap Q^{\prime} \neq \varnothing$ that $Q^{\prime} \subset Q$. If $R \not \subset Q$ then $R^{\prime} \not \subset Q$ and $\mathrm{d}_{\text {tree }}\left(R^{\prime}, Q^{\prime}\right) \geq r+2$ (we need at least one step to go from $Q^{\prime}$ above $Q$, and then at least $r+1$ steps to go to the cubes of size $\left.2^{-r} \ell(R)\right)$. Therefore, $\left\langle T\left(w^{-1} \chi_{Q}\right), h_{R}^{\nu}\right\rangle_{\nu}=0$ if $\ell(R) \leq 2^{-r} \ell(Q)$ and $R \not \subset Q$.

So, the operator $T_{\mu}$ is lower triangularly localized, and the same reasoning can be applied to the adjoint operator $T_{\nu}^{*}$. So we have shown, that under the assumption $u, v \in L_{\text {loc }}^{2}$ the operator $T_{\mu}$ obtained from the band operator $T$ is well localized.

To treat the general case let us note that for $Q \cap R=\varnothing$ the above sum (2.1) has only finitely many terms. Each operator $T_{R^{\prime}, Q^{\prime}}$ is an integral operator with bounded compactly supported kernel, so $\left\langle T \chi_{Q} u, h_{R}^{\nu}\right\rangle_{\nu}$ is well defined for $u, v \in L_{\text {loc }}^{1}$. 
Moreover, if we take increasing sequences of weights $u_{n}, v_{k} \in L_{\text {loc }}^{2}, u_{n} \nearrow u, v_{k} \nearrow v$, and define $d \nu_{k}=v_{k} d m$, then

$$
\lim _{n \rightarrow \infty} \lim _{k \rightarrow \infty}\left\langle T \chi_{Q} u_{n}, h_{R}^{\nu_{k}}\right\rangle_{\nu_{k}}=\left\langle T \chi_{Q} u, h_{R}^{\nu}\right\rangle_{\nu}=\left\langle M_{v^{1 / 2}} T M_{u^{1 / 2}} \chi_{Q} u^{1 / 2}, h_{R}^{\nu} v^{1 / 2}\right\rangle,
$$

where the function $M_{v^{1 / 2}} T M_{u^{1 / 2}} \chi_{Q} u^{1 / 2}$ is interpreted exactly as in the Remark 1.3

2.3. Sawyer type results for well localized operators. The following two theorems can be also considered the main result of the paper.

Theorem 2.2. Let $T=T_{\mu}$ be a well localized operator acting (formally) from $L^{2}(\mu$ ) to $L^{2}(\nu)$. Then $T_{\mu}$ is a bounded operator from $L^{2}(\mu)$ to $L^{2}(\nu)$ if and only if $T$ and its formal adjoint $T_{\nu}^{*}$ are uniformly bounded on characteristic functions of cubes, i.e. iff

$$
\begin{array}{ll}
\left\|T_{\mu} \chi_{Q}\right\|_{L^{2}(\nu)}^{2} \leq C\left\|\chi_{Q}\right\|_{L^{2}(\mu)}^{2}=C \mu(Q), & \forall Q \in \mathcal{D}, \\
\left\|T_{\nu}^{*} \chi_{Q}\right\|_{L^{2}(\mu)}^{2} \leq C\left\|\chi_{Q}\right\|_{L^{2}(\nu)}^{2}=C \nu(Q), & \forall Q \in \mathcal{D} .
\end{array}
$$

Moreover, the norm of $T$ can be estimated by a constant depending only on the dimension $N$, the above constants $C$ and $r$ from the definition of well localized operator.

Theorem 1.2 is an immediate corollary of this theorem.

The assumptions that $T_{\mu}$ and $T_{\nu}^{*}$ are uniformly bounded on the characteristic functions of cubes can be relaxed a little: one does not have to integrate $T_{\mu} \chi_{Q}$ over the whole space. Namely, Theorem 2.2 can be restated as follows

Theorem 2.3. Let $T=T_{\mu}$ be a well localized operator acting (formally) from $L^{2}(\mu$ ) to $L^{2}(\nu)$. Then $T_{\mu}$ is a bounded operator from $L^{2}(\mu)$ to $L^{2}(\nu)$ if and only if the following two conditions hold:

(1) $\left|\left\langle T_{\mu} \chi_{Q}, \chi_{R}\right\rangle_{\nu}\right| \leq C \mu(Q)^{1 / 2} \nu(R)^{1 / 2}$ for all cubes $Q$, R of comparable size, $2^{-r} \ell(Q) \leq \ell(R) \leq 2^{r} \ell(Q)$; here $r$ is the number from the definition of well localized operator.

(2) For all cubes $Q$

$$
\int_{Q}\left|T_{\mu} \chi_{Q}\right|^{2} d \nu \leq C \mu(Q), \quad \int_{Q}\left|T_{\nu}^{*} \chi_{Q}\right|^{2} d \nu \leq C \nu(Q) .
$$

Moreover, the norm of $T$ can be estimated by a constant depending only on the dimension $N$, the above constants $C$ and $r$ from the definition of well localized operator.

Theorem 1.5 is an immediate corollary of the above Theorem 2.3.

Note that in the condition (2) of the theorem one can replace $T_{\mu} \chi_{Q}$ by its orthogonal (in $L^{2}(\nu)$ ) projection onto the subspace of functions $F$ with zero average over $Q, \int_{Q} f d \nu=0$, and similarly for $T_{\nu}^{*} \chi_{Q}$. The condition (1) implies that $\left|\left\langle T_{\mu} \chi_{Q}, \chi_{Q}\right\rangle_{\nu}\right| \leq C(\mu(Q))^{1 / 2}(\nu(Q))^{1 / 2}$, i.e. that the projection onto the orthogonal complement of this subspace is bounded.

While in our main example the measures $\mu$ and $\nu$ are absolutely continuous, and the operator $T$ came from an operator in the non-weighted $L^{2}$, in Theorems 2.2 and 2.3 the measures are arbitrary regular Borel measures and $T_{\mu}$ is an arbitrary operator $L^{2}(\mu) \rightarrow L^{2}(\nu)$. We only need to know its bilinear form $\left\langle T_{\mu} \chi_{Q}, \chi_{R}\right\rangle_{\nu}$ to apply the theorems. 
Remark 2.4. We do not have to assume that the operator $T$ is bounded in the nonweighted $L^{2}$ to be able to apply Theorem 2.2 and 2.3 to get the weighted norm inequalities for $T$. We only need to be able to define $\left\langle T_{\mu} \chi_{Q}, \chi_{R}\right\rangle_{\nu}$. In Section 2.2 above we had shown how one can treat this expression if the operator $T$ is bounded in the non-weighted $L^{2}$. The same reasoning will work if $T$ is bounded in any nonweighted $L^{p}$.

In general situation (for example, if we have unbounded Haar multipliers) some care is needed to interpret $\left\langle T_{\mu} \chi_{Q}, \chi_{R}\right\rangle_{\nu}$, but after that one can apply Theorem 2.2 and 2.3.

\section{Proof of Theorems 2.2 and 2.3.}

3.1. Martingale difference decomposition. Denote by $E_{k}=E_{k}^{\mu}$ the averaging operator in $L^{2}(\mu)$ over dyadic cubes of size (length of the side) $2^{k}$, namely $E_{k}^{\mu} f(x)=$ $\mu(Q)^{-1} \int_{Q} f d \mu$, where $Q$ is a dyadic cube of size $2^{k}$ containing $x$ (for the sake of definiteness, we consider cubes of the form $\left.x_{0}+[a, b)^{N}\right)$. If $Q$ is a cube of size $2^{k}$, we denote by $E_{Q}^{\mu} f$ the restriction of $E_{k}^{\mu} f$ to $Q: E_{Q}^{\mu} f:=\left(\mu(Q)^{-1} \int_{Q} f d \mu\right) \chi_{Q}=\chi_{Q} E_{k}^{\mu} f$.

Let $\Delta_{k}=\Delta_{k}^{\mu}:=E_{k-1}^{\mu}-E_{k}^{\mu}$. Again for a dyadic cube $Q$ of size $2^{k}$, denote by $\Delta_{Q}^{\mu} f$ the restriction of $\Delta_{k}^{\mu} f$ to $Q$. Clearly, for any $f \in L^{2}(\mu)$, the functions $\Delta_{Q}^{\mu} f$, $Q \in \mathcal{D}$, are orthogonal to each other, and that for any fixed $n$ we have the orthogonal decomposition

$$
\begin{aligned}
f & =\sum_{Q \in \mathcal{D}, \ell(Q) \leq 2^{n}} \Delta_{Q}^{\mu} f+\sum_{Q \in \mathcal{D}, \ell(Q)=2^{n}} E_{Q}^{\mu} f, \\
\|f\|_{L^{2}(\mu)}^{2} & =\sum_{Q \in \mathcal{D}, \ell(Q) \leq 2^{n}}\left\|\Delta_{Q}^{\mu} f\right\|^{2}+\sum_{Q \in \mathcal{D}, \ell(Q)=2^{n}}\left\|E_{Q}^{\mu} f\right\|^{2} .
\end{aligned}
$$

3.2. Paraproducts. Define the paraproduct $\Pi^{\mu}=\Pi_{T}^{\mu}$, acting (formally) from $L^{2}(\mu)$ to $L^{2}(\nu)$ by

$$
\Pi^{\mu} f:=\sum_{Q \in \mathcal{D}} E_{Q}^{\mu} f \sum_{\substack{R \in \mathcal{D}, R \subset Q, \ell(R)=2^{-r} \ell(Q)}} \Delta_{R}^{\nu} T_{\mu} \chi_{Q} .
$$

The paraproduct $\Pi^{\nu}=\Pi_{T^{*}}^{\nu}$ is defined similarly,

$$
\Pi^{\nu} f:=\sum_{Q \in \mathcal{D}} E_{Q}^{\nu} f \sum_{\substack{R \in \mathcal{D}, R \subset Q, \ell(R)=2^{-r} \ell(Q)}} \Delta_{R}^{\mu} T_{\nu}^{*} \chi_{Q} .
$$

Remark 3.1. Note, that it follows from the definition of well localized operator that if $R \subset Q$, and $\ell(R) \leq 2^{-r} \ell(Q)$, then for any $Q^{\prime} \supset Q$

$$
\Delta_{R}^{\nu} T_{\mu} \chi_{Q^{\prime}}=\Delta_{R}^{\nu} T_{\mu} \chi_{Q}
$$

In other words, in the definition of $\Pi$ we can always replace $\chi_{Q}$ by $\chi_{Q^{\prime}}$ where $Q^{\prime}$ is a bigger cube.

That essentially means that formally we can write $T 1$ instead of $T \chi_{Q}$, so the definition is more in line with the standard definition of a paraproduct.

The following lemma describes the matrix of $\Pi$ with respect to the weighted Haar systems in $L^{2}(\mu)$ and $L^{2}(\nu)$. 
Lemma 3.2. Let $Q, R$ be dyadic cubes. Then for the paraproduct $\Pi=\Pi^{\mu}$ defined above

(1) If $\ell(R) \geq 2^{-r} \ell(Q)$ then $\left\langle\Pi^{\mu} h_{Q}^{\mu}, h_{R}^{\nu}\right\rangle_{\nu}=0$ for all weighted Haar functions $h_{Q}^{\mu}$ and $h_{R}^{\nu}$.

(2) If $R \not \subset Q$, then $\left\langle\Pi^{\mu} h_{Q}^{\mu}, h_{R}^{\nu}\right\rangle_{\nu}=0$ for all weighted Haar functions $h_{Q}^{\mu}$ and $h_{R}^{\nu}$.

(3) If $\ell(R)<2^{-r} \ell(Q)$, then for all weighted Haar functions $h_{Q}^{\mu}$ and $h_{R}^{\nu}$

$$
\left\langle\Pi^{\mu} h_{Q}^{\mu}, h_{R}^{\nu}\right\rangle_{\nu}=\left\langle T h_{Q}^{\mu}, h_{R}^{\nu}\right\rangle_{\nu}
$$

in particular, if $R \not \subset Q$, then both sides of the equality are 0 .

Proof. Let us use $Q^{\prime}$ and $R^{\prime}$ for the summation indices in the paraproduct, i.e. let us write

$$
\Pi^{\mu} h_{Q}^{\mu}:=\sum_{Q^{\prime} \in \mathcal{D}} E_{Q^{\prime}}^{\mu} h_{Q}^{\mu} \sum_{\begin{array}{c}
R^{\prime} \in \mathcal{D}, R^{\prime} \subset Q^{\prime}, \\
\ell\left(R^{\prime}\right)=2^{-r} \ell\left(Q^{\prime}\right)
\end{array}} \Delta_{R^{\prime}}^{\nu} T_{\mu} \chi_{Q^{\prime}} .
$$

Since $h_{R}^{\nu}$ is orthogonal to ranges of all projections $\Delta_{R^{\prime}}^{\nu}$ except $\Delta_{R}^{\nu}$ we can write

$$
\left\langle\Pi h_{Q}^{\mu}, h_{R}^{\nu}\right\rangle_{\nu}=\left\langle\left(E_{Q^{\prime}}^{\mu} h_{Q}^{\mu}\right) \Delta_{R}^{\nu} T \chi_{Q^{\prime}}, h_{R}^{\nu}\right\rangle_{\nu}=a\left\langle T \chi_{Q^{\prime}}, h_{R}^{\nu}\right\rangle_{\nu}
$$

where $Q^{\prime}$ is the grandparent of $R$ of order $r$ (i.e. the cube $Q^{\prime}, Q^{\prime} \supset R$ and such that $\left.\ell\left(Q^{\prime}\right)=2^{r} \ell(R)\right)$ and $a$ is the value of $E_{Q^{\prime}} h_{Q}^{\mu}$ on $Q^{\prime}, E_{Q^{\prime}} h_{Q}^{\mu}=a \chi_{Q^{\prime}}$.

It is easy to see that $E_{Q^{\prime}}^{\mu} h_{Q}^{\mu} \not \equiv 0$ (equivalently $a \neq 0$ ) only if $Q^{\prime} \varsubsetneqq Q$. Therefore, see (3.2), $\left.\left\langle\Pi h_{Q}^{\mu}, h_{R}^{\nu}\right\rangle_{\nu} \neq\right)$ only if $Q^{\prime} \varsubsetneqq Q$ and statements 1 and 2 of the lemma follow immediately.

Indeed, if $\ell(R) \geq 2^{-r} \ell(Q)$ and $\ell\left(Q^{\prime}\right)=2^{r} \ell(R)$, the inclusion $Q^{\prime} \varsubsetneqq Q$ is impossible, so $\left\langle\Pi h_{Q}^{\mu}, h_{R}^{\nu}\right\rangle_{\nu}=0$, and the statement (1) is proved.

If $R \not \subset Q$, then the inclusion $Q^{\prime} \varsubsetneqq Q$ implies that $R \not \subset Q^{\prime}$. But $\ell(R)=2^{-r} \ell\left(Q^{\prime}\right)$, so by the definition of a well localized operator $\left\langle\Pi h_{Q}^{\mu}, h_{R}^{\nu}\right\rangle_{\nu}=0$ and the statement (2) is proved.

Let us prove statement 3. Let $\ell(R)<2^{-r} \ell(Q)$. If $R \not \subset Q$ then by the statement 2 of the lemma $\left\langle\Pi h_{Q}^{\mu}, h_{R}^{\nu}\right\rangle_{\nu}=0$, and $\left\langle T_{\mu} h_{Q}^{\mu}, h_{R}^{\nu}\right\rangle_{\nu}=0$ by the definition of well localized operators ("children" $Q_{k}$ of $Q$ are cubes of size at least $2^{r} \ell(R)$, and it follows from the definition of well localized operator that $\left.\left\langle T_{\mu} \chi_{Q_{k}}, h_{R}^{\nu}\right\rangle_{\nu}=0\right)$. So, we only need to consider the case $R \subset Q$.

Let $Q_{1}$ be the "child" of $Q$ containing $R$ (i.e. $R \subset Q_{1} \subset Q, \ell\left(Q_{1}\right)=\ell(Q) / 2$ ), and let $a$ be the value of $h_{Q}^{\mu}$ on $Q_{1}$. Then, since for all other children $Q_{k}$ of $Q$ the definition of well localized operator implies $\left\langle T \chi_{Q_{k}}, h_{R}^{\nu}\right\rangle_{\nu}=0$, we can conclude that

$$
\left\langle T h_{Q}^{\mu}, h_{R}^{\nu}\right\rangle_{\nu}=a\left\langle T \chi_{Q_{1}}, h_{R}^{\nu}\right\rangle_{\nu}
$$

On the other hand we have shown before, see (3.2) that

$$
\left\langle\Pi h_{Q}^{\mu}, h_{R}^{\nu}\right\rangle_{\nu}=\left\langle\left(E_{Q^{\prime}}^{\mu} h_{Q}^{\mu}\right) \Delta_{R}^{\nu} T \chi_{Q^{\prime}}, h_{R}^{\nu}\right\rangle_{\nu}
$$

where $R \subset Q^{\prime} \varsubsetneqq Q, \ell\left(Q^{\prime}\right)=2^{r} \ell(R)$. Therefore $Q^{\prime} \subset Q_{1}$ and so $E_{Q^{\prime}}^{\mu} h_{Q}^{\mu}=a \chi_{Q^{\prime}}$. We also know, see Remark 3.1, that because $Q^{\prime} \subset Q_{1}$ we have equality $\Delta_{R}^{\nu} T \chi_{Q^{\prime}}=$ $\Delta_{R}^{\nu} T \chi_{Q_{1}}$. Thus we can continue:

$$
\left\langle\Pi h_{Q}^{\mu}, h_{R}^{\nu}\right\rangle_{\nu}=a\left\langle\Delta_{R}^{\nu} T \chi_{Q^{\prime}}, h_{R}^{\nu}\right\rangle_{\nu}=a\left\langle\Delta_{R}^{\nu} T \chi_{Q_{1}}, h_{R}^{\nu}\right\rangle_{\nu}=a\left\langle T \chi_{Q_{1}}, h_{R}^{\nu}\right\rangle_{\nu} .
$$


Therefore $\left\langle\Pi h_{Q}^{\mu}, h_{R}^{\nu}\right\rangle_{\nu}=\left\langle T h_{Q}^{\mu}, h_{R}^{\nu}\right\rangle_{\nu}$, and the lemma is proved.

It follows from Lemma 3.2 and the definition of a well localized operator, that in the weighted Haar bases the matrix of the difference $T_{\mu}-\Pi_{T}^{\mu}-\left(\Pi_{T^{*}}^{\nu}\right)^{*}$ has finitely many diagonals, which are bounded by the assumption (1) of Theorem 2.3. Therefore, the main part in the proof Theorem 2.3 (and thus Theorem 2.2) is to prove that the paraproducts are bounded. Of course, one also need to take care of the terms like $E_{Q}^{\mu} f$ in the decomposition (3.1), but this is ratther simple, we present the details in Section 3.4 below

3.3. Boundedness of the paraproduct. We will need the following well known theorem. $\mu$.

Let $f_{R}:=\frac{1}{\mu(R)} \int_{R} f d \mu$ be the average of the function $f$ with respect to the measure

Theorem 3.3 (Dyadic Carleson Embedding Theorem). If the numbers $a_{Q} \geq 0$, $Q \in \mathcal{D}$ satisfy the following Carleson measure condition

$$
\sum_{Q \subset R} a_{Q} \leq \mu(R)
$$

then for any $f \in L^{2}(\mu)$

$$
\sum_{R \in \mathcal{D}} a_{R}\left|f_{R}\right|^{2} \leq C\|f\|_{L^{2}(\mu)}^{2}
$$

where $C$ is an absolute constant.

This theorem is very well known, cf [Dur70]. For an alternative prove see also [NTV], [NT], [NTV3], where it was proved with the constant $C=4$ using Bellman function method. It was also proved in [NTV3] that the constant $C=4$ is optimal. We should mention, that in $[\mathrm{NT}]$, [NTV3] this theorem was proved for $\mathbb{R}^{1}$, but the same proof works for general martingale setup. A proof for $\mathbb{R}^{2}$ was presented in [NTV], and the same proof works for $\mathbb{R}^{N}$.

Let us now show that the paraproduct $\Pi=\Pi_{T}^{\mu}$ is bounded. Ranges of the projections $\delta_{R}^{\nu}$ are mutually orthogonal, so to prove the boundedness of the paraproduct $\Pi_{T}^{\mu}$ it is sufficient to show that the numbers

$$
a_{Q}:=\sum_{\substack{R \in \mathcal{D}, R \subset Q \\ \ell(R)=2^{-r} \ell(Q)}}\left\|\Delta_{R}^{\nu} T_{\mu} \chi_{R}\right\|_{L^{2}(\nu)}^{2} \leq
$$

satisfy the Carleson Measure Condition (3.3) from Theorem 3.3. Let us prove this.

Consider a cube $\widetilde{Q}$. We want to show that

$$
\sum_{Q \subset \widetilde{Q}} \sum_{\substack{R \in \mathcal{D}, R \subset Q \\ \ell(R)=2^{-r} \ell(Q)}}\left\|\Delta_{R}^{\nu} T_{\mu} \chi_{Q}\right\|_{L^{2}(\nu)}^{2} \leq C \mu(Q) .
$$

By Remark 3.1 we can replace $\chi_{Q}$ by $\chi_{\widetilde{Q}}$, so the desired estimates becomes

$$
\sum_{\substack{R \in \mathcal{D}, R \subset \widetilde{Q} \\ \ell(R) \leq 2^{-r} \ell(\widetilde{Q})}}\left\|\Delta_{R}^{\nu} T_{\mu} \chi_{\widetilde{Q}}\right\|_{L^{2}(\nu)}^{2} \leq \sum_{R \subset \widetilde{Q}}\left\|\Delta_{R}^{\nu} T_{\mu} \chi_{\widetilde{Q}}\right\|_{L^{2}(\nu)}^{2} \leq\left\|\chi_{\widetilde{Q}} T_{\mu} \chi_{\widetilde{Q}}\right\|_{L^{2}(\nu)}^{2} .
$$


By assumption (2) of Theorem 2.3

$$
\left\|\chi_{\widetilde{Q}} T_{\mu} \chi_{\widetilde{Q}}\right\|_{L^{2}(\nu)}^{2}:=\int_{\widetilde{Q}}\left|T_{\mu} \chi_{\widetilde{Q}}\right|^{2} d \nu \leq C \mu(\widetilde{Q})
$$

and so the sequence $a_{Q}, Q \in \mathcal{D}$ satisfies the condition (3.3)

3.4. Why $T$ is bounded. Let $f \in L^{2}(\mu), g \in L^{2}(\nu)$.

We want to estimate

$$
\left|\left\langle T_{\mu} f, g\right\rangle_{\nu}\right| \leq C\|f\|_{L^{2}(\mu)}\|g\|_{L^{2}(\nu)} .
$$

It is sufficient to prove the estimate on a dense set of compactly supported functions. Each compact subset of $\mathbb{R}^{N}$ is contained in at most $2^{N}$ cubes of the same size as the size of this compact subset, so let $Q_{k}, k=1,2, \ldots, 2^{N}$ be cubes of size $2^{d}$ containing supports of $f$ and $g$.

Let us decompose $f$ and $g$ using the martingale difference decomposition,

$$
f=\sum_{k=1}^{2^{N}} E_{Q_{k}}^{\mu} f+\sum_{Q: \ell(Q) \leq 2^{d}} \Delta_{Q}^{\mu} f, \quad g=\sum_{k=1}^{2^{N}} E_{Q_{k}}^{\nu} g+\sum_{Q: \ell(Q) \leq 2^{d}} \Delta_{Q}^{\nu} g .
$$

Note, that $\Delta_{Q}^{\mu} f$ and $\Delta_{Q}^{\nu} g$ are $\mu$ - and $\nu$-Haar functions on the cube $Q$.

By Lemma 3.2

$$
\begin{aligned}
\left\langle T_{\mu} \sum_{Q} \Delta_{Q}^{\mu} f, \sum_{R} \Delta_{R}^{\nu} g\right\rangle_{\nu}= & \left\langle\Pi_{T}^{\mu} f, g\right\rangle_{\nu}+\left\langle f, \Pi_{T^{*}}^{\nu} g\right\rangle_{\nu} \\
& +\sum_{Q: \ell(Q) \leq 2^{d}}\left(\sum_{R: 2^{-r} \ell(Q) \leq \ell(R) \leq 2^{r} \ell(Q)}\left\langle T_{\mu} \Delta_{Q}^{\mu} f, \Delta_{R}^{\nu} g\right\rangle_{\nu}\right)
\end{aligned}
$$

We know that the paraproducts $\Pi_{T}^{\mu}$ and $\Pi_{T^{*}}^{\nu}$ are bounded, so it remains to estimate the last sum. It follows from the assumption (1) of Theorem 2.3 that

$$
\left|\left\langle T_{\mu} \Delta_{Q}^{\mu} f, \Delta_{R}^{\nu} g\right\rangle_{\nu}\right| \leq C\left\|\Delta_{Q}^{\mu} f\right\|_{L^{2}(\mu)}\left\|\Delta_{R}^{\nu} g\right\|_{L^{2}(\nu)}
$$

if $2^{-r} \ell(Q) \leq \ell(R) \leq 2^{r} \ell(Q)$.

On the other hand, it follows from the definition of a well localized operator, that given a cube $Q$ at most $M$ terms $\left\langle T_{\mu} \Delta_{Q}^{\mu} f, \Delta_{R}^{\nu} g\right\rangle_{\nu}$ are non-zero, where $M$ does not depend on the choice of $Q$. Therefore we can split the sum into at most $M$ sums of form (operators with "one diagonal")

$$
\sum_{Q}\left\langle T_{\mu} \Delta_{Q}^{\mu} f, \Delta_{R(Q)}^{\nu} g\right\rangle_{\nu}
$$

which can be estimated by Cauchy-Schwarz inequality.

It remains to estimate the terms involving $E_{Q}$. By assumption (1) of Theorem 2.3 $\left|\left\langle T_{\mu} E_{Q_{k}}^{\mu} f, E_{Q_{j}}^{\nu} g\right\rangle\right| \leq C\left\|\chi_{Q_{k}}\right\|_{L^{2}(\mu)}\left\|\chi_{Q_{j}}\right\|_{L^{2}(\nu)} \leq C\|f\|_{L^{2}(\mu)}\|g\|_{L^{2}(\nu)}$, and since there are at most $2^{N}$ cubes $Q_{k}$ we can estimate

$$
\sum_{k, j}\left|\left\langle T_{\mu} E_{Q_{k}}^{\mu} f, E_{Q_{j}}^{\nu} g\right\rangle_{\nu}\right| \leq 2^{2 N} C\|f\|_{L^{2}(\mu)}\|g\|_{L^{2}(\nu)}
$$


Let us now estimate

$$
\sum_{k}\left|\sum_{R}\left\langle T_{\mu} E_{Q_{k}}^{\mu} f, \Delta_{R}^{\nu} g\right\rangle_{\nu}\right|
$$

For a fixed $Q_{k}$ we have by the assumption of Theorem 2.2

$$
\left|\sum_{R: \ell(R)<\ell\left(Q_{k}\right)}\left\langle T_{\mu} E_{Q_{k}}^{\mu} f, \Delta_{R}^{\nu} g\right\rangle_{\nu}\right| \leq C\|f\|_{L^{2}(\mu)}\|g\|_{L^{2}(\nu)} .
$$

But we have at most $2^{N}$ cubes $Q_{k}$, so the whole sum is bounded by

$$
2^{N} C\|f\|_{L^{2}(\mu)}\|g\|_{L^{2}(\nu)} .
$$

The sum

$$
\sum_{k}\left|\sum_{R}\left\langle T_{\mu} \Delta_{R}^{\mu} f, E_{Q_{k}}^{\nu} g\right\rangle_{\nu}\right|
$$

can be estimated similarly.

\section{References}

[CS1] M. Cotlar, C. Sadosky, On the Helson-Szegö theorem and a related class of modified Toeplitz kernels, in Harmonic Analysis in Euclidean apaces, ed. by G.Weiss and S. Wainger, Proc. Symp. Pure Math. 35, Amer. Math. Soc., Providence, R.I., 1979, 383-407.

[CS2] M. Cotlar, C. Sadosky, On some $L^{p}$ version of the Helson-Szegö theorem, Conference on Harmonic Analysis in honor of Antony Zygmund (Chicago, 1981), vol.1, ed. by W. Beckner et al., Wadsworth Math. Ser,. Wadsworth, Belmont, CA, 1983, 306-317.

[ChWW] A. Chang, J.M. Wilson, Th. Wolff, Some weighted norm inequalities concerning the Schrädinger operators, Comment. Math. Helvetici, 60 (1985), 217-246.

[Dur70] P. L. Duren, Theory of $H^{p}$ spaces, Pure and Applied Mathematics, Vol. 38, Academic Press, New York, 1970.

[FKP] R.A. Fefferman, C.E. Kenig, J. Pipher, The theory of weights and the Dirichlet problem for elliptic equations, Ann. of Math. 134 (1991), 65-124.

[G] J. B. Garnett, Bounded analytic functions. Pure and Applied Mathematics, 96. Academic Press, Inc. [Harcourt Brace Jovanovich, Publishers], New York-London, 1981. xvi+467

[KV] N.J. Kalton, I.E. Verbitsky, Nonlinear equations and weighted norm inequalities, Trans. Amer. Math. Soc. 351 (1999), no. 9, 3441-3497

[NT] F. L. Nazarov and S. R. Treil, The hunt for a Bellman function: applications to estimates for singular integral operators and to other classical problems of harmonic analysis, Algebra i Analiz 8 (1996), no. 5, 32-162.

[NTV] F. Nazarov, S. Treil and A. Volberg, Cauchy integral and Calder'on-Zygmund operators on non-homogeneous spaces, IMRN (Int. Math.Res. Notes.), 1997, No. 15, 703-726.

[NTV1] _ The Bellman function and two weight inequalities for Haar multipliers, Journal of the American Mathematical Society, 12(1999), no. 4, 909-928.

[NTV2] _ The Tb-theorem on non-homogeneous spaces, Acta Mathematica, 190 (2003), 151239.

[NTV3] _ Bellman function in stochastic control and harmonic analysis, Systems, approximation, singular integral operators, and related topics (Bordeaux, 2000), 393-423, Oper. Theory Adv. Appl., 129, Birkhtuser, Basel, 2001.

[NTV4] _ Two weight estimate for the Hilbert transform, Preprint, 2004, pp. 1-40.

[NV] F. Nazarov, A. Volberg, Bellman function, two weight Hilbert transform and imbedding for the model space $K_{\theta}$, Volume in the memory of Tom Wolff. J. d'Analyse Math., 87, (2003), 385-414.

[P] St. Petermichl, Dyadic shifts and a logarithmic estimate for Hankel operators with matrix symbol, C. R. Acad. Sci. Paris Sé r. I Math. 330 (2000), no. 6, 455-460. 
[PTV] St. Petermichl, S. Treil and A. Volberg, Why the Riesz transforms are averages of the dyadic shifts?, Proceedings of the 6th International Conference on Harmonic Analysis and Partial Differential Equations (El Escorial, 2000). Publ. Mat. 2002, Vol. Extra, 209-228.

[S] C. Sadosky, Lifting of kernels shift-invariant in scattering systems, Holomorphic spaces, MSRI publications, 33, 1998.

[S1] E.T. Sawyer, A characterization of a two weight norm inequality for maximal operators, Studia Math. 75 (1982), 1-11.

[S2] - A characterization of two weight norm inequality for fractional and Poisson integrals, Trans. Amer. Math. Soc. 308 (1988), 533-545.

[SW] E.T. Sawyer, R.L. Wheeden, Weighted inequalities for fractional integrals on Euclidean and homogeneous spaces, Amer. J. Math. 114 (1992), 813-874.

[T] X. Tolsa, Boundedness of the Cauchy integral operator, Preprint,1997.

[TV1] S. Treil and A. Volberg, Wavelets and the angle between past and future, J. Funct. Anal. 143 (1997), no. 2, 269-308.

[TV2] Weighted embeddings and weighted norm inequalities for Hilbert transform and maximal operator, St. Petersburg Math. J. 7 (1996), 207-226.

[VW] I.E. Verbitsky, R.L. Wheeden, Weighted norm inequalities for integral operators, Trans. Amer. Math. Soc. 350 (1998), no. 8, 3371-3391.

[V] A.Volberg, Matrix $A_{p}$ weights via S-functions, J. Amer. Math. Soc. 10 (1997), 223-246.

Department of Mathematics, Michigan State university, East Lansing, Mi, 48824

E-mail address: fedja@math.msu.edu

Current address: Mathematics Department, University of Wisconsin-Madison, 480 Lincoln Dr Madison WI 53706-1388

E-mail address: nazarov@math.wisc.edu

Department of Mathematics, Brown University, Box 1917, 151 Thayer Street, ProviDENCE, RI 02912, USA

E-mail address: treil@math.brown.edu

Department of Mathematics, Michigan State university, East Lansing, Mi, 48824

E-mail address: volberg@math.msu.edu 